\title{
«VERGILIUS NON ERO, MARSUS ERO»: LA FIGURA Di Domizio Marso Nell'opera di MarZIALE
}

\section{Abstract}

Il contributo si propone di indagare la presenza della figura di Domizio Marso all'interno dell'opera di Marziale, avanzando delle ipotesi in merito al ruolo di mediazione esercitato dal poeta augusteo nella storia dell'epigramma latino. Le frequenti e significative menzioni nel corpus marzialiano, che evidenziano una progressiva identificazione di Marziale con il suo antecedente, suggeriscono che Marso, prendendo le mosse dal modello catulliano, non abbia esitato a ricercare soluzioni poetiche nuove e originali, facendosi autore di una poesia improntata a un forte sperimentalismo, nelle forme e nei contenuti: fu ora poeta leggero e ora impegnato, rappresentando per Marziale un esplicito punto di riferimento, sia nell'interpretazione fluida e aperta del genere epigrammatico, sia per la capacità di comporre un epigramma degno, non meno della poesia "maggiore", di adempiere alla celebrazione del potere. È possibile ipotizzare, inoltre, che Marso abbia offerto un rilevante contributo, in particolare, nello sviluppo dell'epigramma longum, discostandosi dal principio callimacheo della brevitas ma pur sempre mantenendosi fedele alla sua "Musa minore".

The contribution aims to investigate the presence of Domitius Marsus within Martial's work, advancing hypotheses about the role of mediation held by the Augustan poet in the history of the Latin epigram. Martial's corpus often includes significant quotes highlighting Martial's progressive identification with his predecessor, suggesting that Marsus did not hesitate to look for unique new poetic solutions. As a result, his poetry was highly experimental, in terms of both form and content: alternating between a lighter approach and a more serious, committed one, Marsus represented a clear reference point for Martial, both for his fluid and open interpretation of the epigrammatic genre, as well as for his ability to compose an epigram worthy to fulfil the celebration of power, just like "major" poetry. It could also be argued that Marsus offered a considerable contribution particularly to the development of the epigramma longum, moving away from the callimachean principle of brevitas while remaining faithful to his "minor Muse".

Del poeta augusteo Domizio Marso possediamo soltanto pochi frammenti, ${ }^{1}$ che ci consentono di tratteggiare una figura dai contorni alquanto sfumati: vicino ad Augusto $^{2} \mathrm{e}$

\footnotetext{
1 La bibliografia su Domizio Marso è piuttosto ampia e si concentra in particolare negli anni che seguirono la scoperta degli Epigrammata Bobiensia, la cui editio princeps è pubblicata da MUNARI 1955. Tra gli studi complessivi più significativi, si vedano: BARDON 1956, pp. 52-67; Alfonsi 1964, pp. 377 388; Fogazza 1981; Duret 1983, pp. 1480-1487; Courtney 1993, p. 300 e ss.; Hollis 2007, p. 300 e ss.; HeNRIKSÉn 2019, p. 461 e ss., cui rimando per indicazioni bibliografiche aggiornate.

2 Il legame di Marso con Augusto è suffragato, oltre che dagli epigrammi in onore di Azia (frr. 8-9 Blänsdorf $=$ Courtney $=181-182$ Hollis $)$, anche dalla testimonianza di Quint. inst. 3, 1, 18, secondo cui Marso sarebbe stato, insieme al futuro princeps, allievo di Apollodoro di Pergamo. Inoltre, sono
} 
all'entourage mecenaziano, ${ }^{3}$ Marso doveva essere apprezzato dai contemporanei in modo particolare per i suoi epigrammi, del cui carattere sagace e velenoso, ${ }^{4}$ tuttavia, non possiamo avere che una vaga impressione, suggerita dai pochi versi giunti sino a noi e dalle altrettanto scarne testimonianze antiche.

Se quello di Domizio Marso è per noi poco più che un nome, un personaggio senza volto nella fitta schiera degli autori minori di età augustea, così non doveva essere non solo agli occhi dei contemporanei ma anche di Marziale che, nella sua opera, fa riferimento a Marso in ben otto occasioni, un numero molto significativo soprattutto se rapportato a un totale di poco più di una decina di menzioni nell'intera letteratura latina. ${ }^{5}$ Una presenza così diffusa nell'opera marzialiana è da ricondursi presumibilmente all'importante ruolo di mediazione che Marso avrebbe rivestito tra l'epigramma di Catullo e quello di Marziale. ${ }^{6}$

attestati legami familiari fra Ottaviano e la gens Domitia, come nel caso del matrimonio di Lucio Domizio Enobarbo, console nel 16 a.C., con Antonia Maggiore, figlia di Marco Antonio e nipote di Augusto. Per alcune ipotesi sull'origine familiare di Marso e su altre relazioni tra i Domizi e Ottaviano si veda PASETTO - SANSONE 2016.

3 L'appartenenza al circolo di Mecenate, postulata sulla base di Mart. 7, 29 e 8, 55 (56), è recentemente messa in dubbio da BYRNE 2004 e riaffermata in modo convincente da NAUTA 2007, p. 11 e ss.

4 Il noto fr. 1 Blänsdorf (= Courtney $=174$ Hollis), tradito da Filargirio nel suo commento a Verg. Ecl. 3, $90(65 \mathrm{H})$, è tratto dalla raccolta Cicuta. Il titolo è interpretato per primo da HAVET 1900, p. 91 in riferimento al carattere velenoso dei componimenti: «c'est dans l'anecdote que doit résider le venin ou le poison de l'épigramme; nous pourrison dire le vitriol, en style modern, en style antique, Domitius a caractérisé l'épigramme par le titre même de tout le livre: la Ciguë». L'ipotesi è oggi accolta dalla maggior parte degli studiosi, quali ad esempio Fogazza 1981, p. 18; CourTNEY 1993, p. 301; Hollis 2007, pp. 306-307. È ormai pressoché abbandonata la teoria poco persuasiva, sostenuta da RIBBECK 1889, p. 364; Hubaux 1930, pp. 86-87 e PARATORE 1964, p. 77, secondo cui cicuta sarebbe da intendersi piuttosto quale sinonimo di fistula, calamus, quindi come una possibile allusione al genere bucolico (vd. contra MARIOTTI 1963, p. 601 nt. 40).

5 Oltre ai riferimenti marzialiani, Marso è citato in Ov. Pont. 4, 16, 1-6; Plin. nat. 1, 34 ind. auct.; Quint. inst. 3, 1, 18 e 6, 3, 102 e ss. Dallo stesso Marziale dipende quasi sicuramente la testimonianza di Sidonio Apollinare (carm. 9, 259-260: non Gaetulicus hic tibi legetur, / non Marsus, Pedo, Silius, Tibullus), su cui si veda SQuillante Saccone 2009, p. 147 e ss.

6 Sul ruolo di mediazione esercitato da Marso fra l'epigramma di Catullo e quello di Marziale, si veda Citroni 1991, p. 182: «Marziale poteva trovare in lui qualcosa che non trovava in Catullo: non solo la compresenza [...] di epigramma scommatico e celebrativo, non solo un aperto ricondursi alle forme più proprie della tradizione epigrammatica, ma probabilmente anche un interesse più sistematico per il gioco epigrammatico in quanto tale, per le sue tecniche e per i vari aspetti della comicità verbale, non necessariamente carica della tensione lirico-soggettiva di Catullo». Significativa la distanza rispetto al poeta neoterico anche dal punto di vista del destinatario: se la poesia catulliana nasce e si sviluppa all'interno della ristretta cerchia degli homines venustiores, mentre quella di Marziale mira a raggiungere il pubblico eterogeneo del "lettore comune", possiamo affermare che Marso rappresenti, ancora una volta, un passaggio intermedio (cfr. Citroni 1995, pp. 480-482). Il pubblico al quale si rivolge è costituito dalla corte augustea e da Augusto stesso, la cui celebrazione doveva certo avere un ruolo di rilievo all'interno della produzione poetica di Marso. Non bisogna dimenticare, infine, che "Catullus is not easily categorized [...]. Even though the idea that Catullus should be accepted as an epigrammatist was not unknown [...], his other roles were more dominant» (Swann 1994, p. 4). Marso è, al contrario, molto più facile da inquadrare come modello fondamentale per il solo genere epigrammatico. Per tutte queste ragioni, Marziale non arriverà mai a una completa identificazione con Catullo (modello 
Il presente contributo si propone di analizzare i passi del corpus marzialiano nei quali ricorre il nome di Domizio Marso, evidenziando un'evoluzione nel rapporto di Marziale con il suo modello, nella direzione di una progressiva identificazione con il poeta augusteo. Si ipotizzerà, inoltre, un possibile ruolo di Marso nello sviluppo della tipologia epigrammatica dell'epigramma longum.

È sicuramente significativo che Marso compaia per la prima volta in un contesto di particolare rilievo, ossia l'epistola prefatoria al primo libro:

Lascivam verborum veritatem, id est epigrammaton linguam, excusarem, si meum esset exemplum: sic scribit Catullus, sic Marsus, sic Pedo, sic Gaetulicus, sic quicumque perlegitur. ${ }^{7}$

L'autore, prima ancora di cominciare, intende giustificarsi per la lasciva verborum veritas dei suoi componimenti, identificando in Catullo, Marso, Pedone e Getulico i propri modelli. Marziale delinea, in tal modo, una rapidissima storia dell'epigramma latino, inserendosi orgogliosamente in una ben precisa tradizione letteraria, ${ }^{8}$ che possiamo, purtroppo, ricostruire solo in minima parte: di Albinovano Pedone ${ }^{9}$ e di Gneo Cornelio Lentulo Getulico, ${ }^{10}$ infatti, non possediamo neppure un verso epigrammatico, mentre nel caso di Domizio Marso, come si è detto, le testimonianze sono troppo poche perché se ne possa comprendere pienamente l'importanza.

Incontriamo di nuovo il nostro poeta nell' epigramma 2, 71, ancora una volta in coppia con Catullo:

\section{Candidius nihil est te, Caeciliane. Notavi,}

difficilmente eguagliabile, se non altro per fama), mentre il legame con Marso diverrà, nel corso della raccolta, sempre più stretto, fino a culminare, a 8,55 (56), nel «potentissimo simbolo enniano della reincarnazione: Marsus ero» (CANOBBIO 2005, p. 151).

7 Gli epigrammi di Marziale sono riportati secondo l'edizione di Shackleton Bailey 1993.

8 Per una sintetica ricostruzione della storia dell'epigramma latino, si veda CITRONI 2015, pp. 76-85.

9 Albinovano Pedone, identificabile con il prefetto di cavalleria nominato da Tacito in ann. 1, 60, 2, fu autore di epigrammi e di una Teseide, come attesta Ov. Pont. 4, 10, 71-74. È noto in modo particolare, tuttavia, per un poema storico, di cui Seneca il Vecchio in suas. 1,15 ci ha consegnato ventitré versi (fr. 1 Blänsdorf $=$ Courtney $=228$ Hollis), relativi alla navigazione della flotta romana sul Mare del Nord, al ritorno da una spedizione guidata da Germanico. Tra i contributi più significativi, si vedano BARDON 1956, pp. 69-73; TANDOI 1964 e 1967; Duret 1983, pp. 1496-1501; Courtney 1993, p. 315 e ss.; HolLIS 2007 , p. 372 e ss. Per la vasta bibliografia relativa al v. 19, che TrAINA 1985, p. 111 definì «la più celebre crux della letteratura latina», rimando alle indicazioni fornite da MAzzoLI 2002, pp. 167-177. 10 Gneo Cornelio Lentulo Getulico, console ordinario nel 26 d.C., è menzionato da Plin. epist. 5, 3, 5 e da Sidon. epist. 2, 10, 6 come poeta d'amore. Dopo aver esercitato il comando sulle legioni nella Germania Superiore sotto Tiberio, fu condannato a morte per ordine di Caligola nel 39 d.C., «one of the most confused periods of the Julio-Claudian era» (BARRETT 1996, p. 60). Sulle discusse motivazioni alla base della condanna, si veda, con indicazioni bibliografiche, BARZANò 2011, pp. 65-80. Dell'opera di Getulico possediamo soltanto tre esametri di contenuto astronomico (fr. 1 Blänsdorf = Courtney), traditi da Probo ad Verg. Georg. 1, 227, su cui si vedano Dahlmann 1979, pp. 657-667; Traglia 1988; Courtney 1993, pp. 345-346; Merli - Romano 2017, p. 42 e ss. Sulla possibile identificazione

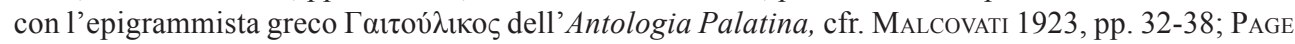
1981, pp. 49-60; HeNRIKSÉn 2019, p. 460; contra DuREt 1986, p. 3172. 


\begin{abstract}
si quando ex nostris disticha pauca lego, protinus aut Marsi recitas aut scripta Catulli.

Hoc mihi das, tamquam deteriora legas,

5 ut collata magis placeant mea? Credimus istud:

malo tamen recites, Caeciliane, tua.
\end{abstract}

Il destinatario, Ceciliano, è definito ironicamente candidus, anche se non perde occasione per far sentire in soggezione il poeta, confrontando i suoi versi con quelli di Marso e di Catullo. L'epigramma si conclude con la replica di Marziale: malo tamen recites, Caeciliane, tua. In questo modo, dal confronto con i pessimi versi di Ceciliano, risalterebbe senz'altro la migliore qualità di quelli di Marziale. Al di là della polemica nei confronti di un detrattore, in questo componimento è ancora evidente la percezione, da parte di Marziale, della propria distanza rispetto ai grandi modelli del passato.

Poco più avanti, nel carme 77 dello stesso libro, l'autore chiama di nuovo in causa Marso, per giustificarsi di fronte a quanti criticavano la lunghezza dei suoi epigrammi:

\author{
Cosconi, qui longa putas epigrammata nostra \\ utilis unguendis axibus esse potes. \\ Hac tu credideris longum ratione colosson \\ et puerum Bruti dixeris esse brevem. \\ 5 Disce quod ignoras: Marsi doctique Pedonis \\ saepe duplex unum pagina tractat opus. \\ Non sunt longa quibus nihil est quod demere possis, \\ sed tu, Cosconi, disticha longa facis.
}

Spesso Marso e il dotto Pedone hanno composto epigrammi addirittura di due "pagine"." Non sono troppo lunghi i componimenti ai quali non si possa togliere nulla, proprio come non è troppo grande il Colosso di Rodi. Sono lunghi, piuttosto, i distici scadenti di Cosconio.

Con questi versi programmatici, Marziale rivendica il diritto a scrivere epigrammata longa ${ }^{12}$ alternandoli, secondo il proprio gusto, a componimenti brevi o brevissimi, come sono, non a caso, quelli che seguono. ${ }^{13}$

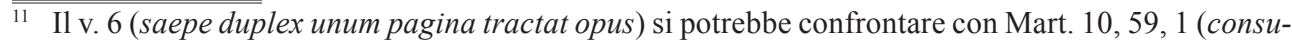
mpta est uno si lemmate pagina, transis), che sarà discusso tra poco a testo: in entrambi i casi, infatti, l'autore cerca di quantificare la lunghezza degli epigrammi, che sono longa in quanto non si estendono soltanto per pochi versi, ma per una "pagina" intera o, addirittura, due. Il termine pagina si riferisce con buona probabilità a una colonna del volumen papiraceo: considerando che una colonna era composta generalmente da un numero di righe compreso tra 25 e 45, un epigramma di due intere colonne (quindi di più di 50 vv.) sarebbe stato senza dubbio molto più esteso della norma (cfr. WiLLiams 2004, p. 243). 12 Riprendo la definizione di epigramma longum che dà CANoBbio 2008, p. 173: «Sulla scorta [...] dei quattro epigrammi apologetici $(1,110 ; 3,83 ; 6,65 ; 8,29)$, ritengo che si possa individuare nei ventidue versi dell'epigramma 8,28 - il più breve tra quelli che Marziale si premura di difendere - la dimensione minima autorialmente garantita dell'epigramma longum marzialiano, che quindi, accettando questo criterio, risulterebbe rappresentato da trentuno testi». Come osserva MorelLi 2008a, p. 23, gli epigrammata longa, così identificati, costituiscono l'1,22\% dei componimenti raccolti nell'opera di Marziale. Vi sono però anche differenti proposte relative alla definizione di questa tipologia epigrammatica, che molti studiosi estendono ai componimenti dai 15 versi in su (sulla base di Szelest 1980, p. 99), per cui rimando ancora alla trattazione di MoReLLi 2008a, pp. 25-28.

13 Merli 2015, p. 279 nt. 85.
} 
Una polemica piuttosto simile nei confronti del lettore gulosus, cui breviora, non meliora placent, si ritrova nell'epigramma 10, 59:

Consumpta est uno si lemmate pagina, transis, et breviora tibi, non meliora placent.

Dives et ex omni posita est instructa macello cena tibi, sed te mattea sola iuvat.

$5 \quad$ Non opus est nobis nimium lectore guloso; hunc volo, non fiat qui sine pane satur.

Di una sontuosa cena bisogna essere in grado di apprezzare tutte le portate, non soltanto gli sfiziosi manicaretti. Il lettore, allo stesso modo, deve saper cogliere l'opera letteraria nella sua interezza, non godendo soltanto dei componimenti più brevi e piacevoli e tralasciando quelli che occupano "un'intera pagina". È evidente, in concomitanza con la sempre maggiore consapevolezza della dignità letteraria dei propri ver$\mathrm{si}^{14}{ }^{14}$ la distanza rispetto alle prime raccolte, nelle quali l'autore invitava umilmente il suo pubblico a una lettura selettiva degli epigrammi.

Coerentemente con la scelta dichiarata di collocarsi nel solco della tradizione epigrammatica latina, Marziale prende dunque le distanze dalla rigida condanna callimachea del $\mu \varepsilon ́ \gamma \alpha \beta i \beta \lambda$ íov, per incontrare piuttosto il gusto catulliano per la varietas, sostenendo l'assoluta libertà dell'autore nella sperimentazione di stili poetici differenti. ${ }^{15}$

Non è casuale che, nella difesa dell' epigramma longum, Marso sia citato insieme a Pedone, piuttosto che a Catullo: con le parziali eccezioni dei carmi 76 e 99, infatti, non sono attestati in Catullo epigrammi di più di venti versi, ${ }^{16}$ del tutto assenti, per altro, anche nella tradizione greca. ${ }^{17}$ Possiamo dedurne che l'epigramma longum abbia avuto origine in ambito romano e in un momento successivo alla pubblicazione del Liber catulliano. Per quanto i frammenti in nostro possesso non ci diano alcuna conferma in tal senso, è ragionevole ipotizzare che qualche autore di età augustea (forse proprio Domizio Marso) abbia raccolto l'eredità catulliana, innovando il genere dell'epigramma latino fino a condurlo, per estensione e forse anche per contenuto, come vedremo, a stretto contatto con altri generi letterari, come l'elegia e la lirica.

Anche l'epigramma 4, 29 contiene un riferimento alla lunghezza dell'opera di Marso:

Obstat, care Pudens, nostris sua turba libellis lectoremque frequens lassat et implet opus.

Rara iuvant: primis sic maior gratia pomis,

\footnotetext{
$\overline{\overline{14} \text { Cfr. Citroni 1991 }}$, p. 186: «Nella maturità della sua esperienza di poeta Marziale rifiuterà ormai apertamente per la sua opera una pura finalità di intrattenimento piacevole o piccante: la sua ambizione è di offrire vera sostanza di contenuto poetico».

15 Cfr. Canobbio 2008, p. 179 e ss.

16 Per un'analisi approfondita degli epigrammi 76 e 99 di Catullo, nati da una raffinata fusione di suggestioni e tradizioni letterarie differenti, si veda MoreLLi 2008b, p. 84 e ss.

17 L'indipendenza della tradizione epigrammatica latina da quella greca, evidenziata dalla presenza di due differenti archetipi, rispettivamente Catullo e Callimaco, è argomentata da Citroni 2004, pp. 127-130.
} 
hibernae pretium sic meruere rosae;
$5 \quad$ sic spoliatricem commendat fastus amicam
ianua nec iuvenem semper aperta tenet.
Saepius in libro numeratur Persius uno
quam levis in tota Marsus Amazonide.
Tu quoque de nostris releges quemcumque libellis,
esse puta solum: sic tibi pluris erit.

Marziale si rivolge all'amico Pudente, forse in risposta all'invito a pubblicare nuovi epigrammi, ${ }^{18}$ esprimendo la preoccupazione che un'opera troppo estesa finisca per annoiare il lettore. Il concetto è ribadito, nei versi seguenti, dal principio più ampio secondo cui, in generale, rara iuvant: si apprezzano in modo particolare le primizie dei campi, o le rose che sbocciano in inverno, così come non è una porta sempre aperta ma un'amante capricciosa e ritrosa a far impazzire i giovani. Allo stesso modo, è stato di gran lunga maggiore il successo di un solo libro di satire di Persio, ${ }^{19}$ piuttosto che dell'intera Amazonis di Domizio Marso, che possiamo quindi dedurre fosse un'opera articolata in più libri. Per questo l'autore invita l'amico ad apprezzare ciascuno dei suoi libelli come se fosse l'unico.

È possibile cogliere, in questi versi, una polemica forse assimilabile a quella presente nel già citato epigramma 10, 59: nel mirino di Marziale c'è, ancora una volta, il lector gulosus, colui che mostra di gradire soltanto i piatti più elaborati, ignorando il resto di un lauto banchetto, che finisce per stancarlo e "saziarlo" (v. 2: lectoremque frequens lassat et implet opus), o che valuta un'opera letteraria solo in merito all'aderenza al principio alessandrino della brevitas.

L'interpretazione dell'epigramma è piuttosto controversa, innanzitutto per quanto riguarda il significato da attribuire all'aggettivo levis: gli studiosi sembrano per lo più escludere che Marziale lo abbia utilizzato in senso spregiativo, con il valore di "superficiale", "vuoto" o "frivolo". ${ }^{20}$ Sembra chiaro, invece, l'impiego del termine in riferimento al genere letterario praticato da Marso, "leggero", "tenue", "giocoso", tutt'altro che gravis. A partire dall'età augustea, infatti, l'aggettivo ricorre molto frequentemente in sede proemiale o programmatica per esplicitare l'adesione a un genere minore, spesso contrapposto alla letteratura maggiore. ${ }^{21}$ Lo stesso Marziale impiega l'aggettivo in altre due occasioni, ora per designare il contenuto scherzoso e a tratti osceno del settimo libro dei suoi epigrammi $(7,8,9$ : fas audire iocos levioraque carmina, Caesar), ora in relazione al genere elegiaco (12, 94, 8: ludo levis elegos).

Accogliendo questo più probabile valore di levis, rimane in dubbio il giudizio di Marziale nei confronti della perduta Amazonis. Secondo Merli, lo stile di Marso sareb-

\footnotetext{
$\overline{18}$ Per un commento all'epigramma 4, 29 rimando a Moreno Soldevila 2006, pp. 253-257.

19 Un riferimento analogo all'opera di Persio si trova negli stessi anni in Quint. inst. 10, 1, 94: Multum et verae gloriae quamvis uno libro Persius meruit. L'accento è posto, proprio come nel v. 7 dell'epigramma di Marziale, sul fatto che l'opera di Persio fosse composta da un solo fortunatissimo libro. 20 Questa era l'interpretazione, ad esempio, di Haupt 1876, p. 333 e Rostagni 1964, II, p. 274.

21 Sul valore di levis nel dibattito critico-letterario romano, si veda BorGo 2003 (in particolare, sul significato dispregiativo del termine, p. 4; sull'impiego nell'ambito della poesia minore e in Marziale, pp. 5-10).
} 
be stato «"giocoso", "allegro", adatto cioè al genere epigrammatico e non all'epica»,22 similmente Hollis afferma che «as an epigrammatist, Martial rated Domitius Marsus highly; so levis here, as well as indicating a lack of epic gravitas, shows what Marsus did better, perhaps referring specifically to the Cicuta, for which this epithet would be appropriate». ${ }^{23}$ Marso, dunque, sarebbe stato senza alcun dubbio levis nella sua produzione epigrammatica, ma di certo non nella prolissa e poco riuscita Amazonis, verso la quale sarebbe implicito un giudizio negativo di Marziale. Opposta la lettura di Fogazza, secondo cui, al contrario, l'aggettivo levis connoterebbe in modo molto positivo il "piacevole" Marso contrapponendolo al "noioso" Persio. ${ }^{24}$

Non credo che, nell'epigramma marzialiano, sia necessariamente presente un confronto fra Marso e Persio sulla base di un giudizio di valore: Marziale non sembra preferire un autore all'altro, ma sembra piuttosto affermare che la lunghezza o la brevità non siano criteri sufficienti per valutare la qualità di un prodotto letterario. Non è detto che un'opera sia migliore di un'altra solo perché brevis, né che la si debba liquidare con scarso interesse solo perché troppo lunga, dato che non sunt longa quibus nihil est quod demere possis. Il timore di Marziale è quello di fare la stessa fine di Marso, ${ }^{25}$ la cui opera fu accolta in modo a dir poco tiepido dal pubblico dei lettori, che non ne notarono la levitas ma soltanto l'impegnativa mole.

Si è osservata, inoltre, una suggestiva affinità fra i vv. 7-8 dell'epigramma e il prologo degli Aitia di Callimaco:26

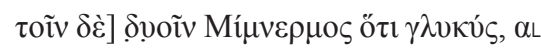

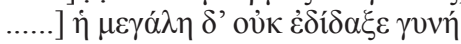

Che Mimnermo sia dolce, fra le due opere,...non lo ha insegnato la grande donna. ${ }^{27}$

22 MerLi 2015, pp. 382-383, nt. 32.

23 Hollis 2007, p. 306; simili interpretazioni del passo marzialiano si trovano già in WeICHERT 1830, pp. 256-257; BARDON 1956, p. 57; MARIOTTI 1963, p. 612.

24 Fogazza 1981, p. 23. Anche Lombardi 1977, p. 356 e ntt. 44-45 propone di cogliere, nelle parole di Marziale, una contrapposizione fra Marso e Persio e, in particolare, fra lo stile «armonioso, scorrevole, senz'asperità» del primo e quello complesso e retorico del secondo. A sostegno di quest'ipotesi si potrebbe citare la testimonianza, tarda ma significativamente ispirata all'opera marzialiana (cfr. nt. 5), di Sidon. carm. 9, 263 (non Persi rigor aut lepos Properti), su cui si veda, anche per indicazioni bibliografiche, Formicola 2009, pp. 86-87. La contrapposizione fra lepos e rigor potrebbe forse fornire una chiave interpretativa valida anche per l'epigramma 4, 29 di Marziale.

25 Come evidenzia già BARDON 1956, p. 57.

26 L'analogia fra i due passi è notata da CAMERON 1995, pp. 311-312: «Editors have rightly been surprised that Martial should pick Marsus "as a typical long-winded epicist". The answer is that, like Callimachus, he picked an elegiac predecessor who for all his virtues was less than concise. There was no epic Amazonis; Martial is simply describing Marsus's elegies on the "dusky Melaenis" in Callimachean terms as a big lady - like the Smyrneis an Amazon». Se l'accostamento fra l'epigramma di Marziale e il frammento callimacheo risulta convincente (tanto che sorprende lo scarso interesse con cui HoLLIS 2007, p. 306 liquida l'ipotesi), non è forse altrettanto accettabile l'identificazione, piuttosto arbitraria, dell'Amazonis con la fusca Melaenis del componimento 7, 29.

27 I vv. 11-12 del fr. 1 Pf. sono molto dibattuti dal punto di vista filologico. Per una recente disamina della questione, con analisi delle diverse proposte di integrazione, si veda CASANOva 2015, p. 143

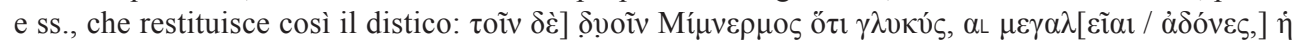


Nel celebre attacco contro gli odiati Telchini, Callimaco difende la sua propensione per

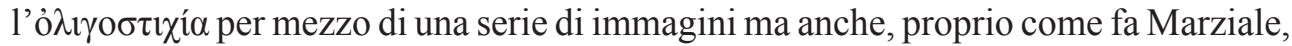
riferendosi esplicitamente a poeti illustri, Filita di Cos e Mimnermo, apprezzati per i loro componimenti brevi piuttosto che per le opere ampie e impegnative. È probabile

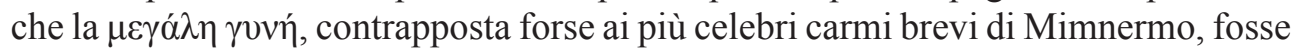
identificabile con la Smirneide, raccolta elegiaca dedicata all'Amazzone eponima di Smirne. ${ }^{28}$ La "pesantezza" di quest'ultima opera avrebbe del tutto soffocato la levitas del grande poeta greco.

Non è improbabile che Marziale, nell'epigramma 4, 29, intendesse riecheggiare proprio questi famosi versi callimachei, seppure con un intento diverso: dal suo punto

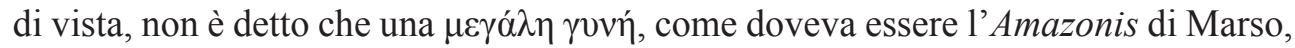
non possa essere anche levis, coniugando la sua possente mole con un'innata eleganza. Marziale intenderebbe in tal modo superare i limiti imposti dalla rigida poetica di Callimaco, per rivendicare ancora una volta il diritto dell'autore a scrivere trascendendo il vincolo della brevitas, che certo non può essere l'unico metro di valutazione di un'opera d'arte. ${ }^{29}$ Altrimenti, come abbiamo letto a 2, 77, si finirebbe per giudicare troppo grande persino il Colosso di Rodi.

L'epigramma di Marziale è, di fatto, l'unica fonte in nostro possesso relativa all' $A$ mazonis di Marso: non disponendo di ulteriori informazioni, è forse impossibile stabilire con certezza a quale genere letterario appartenesse. L'unico punto fermo sembrerebbe essere il fatto che si trattasse di un'opera in più libri. L'ipotesi più diffusa è che fosse un poema epico, ${ }^{30}$ al quale l'autore si sarebbe accostato nella fase più matura della sua produzione letteraria e che si sarebbe rivelato un clamoroso fiasco, un

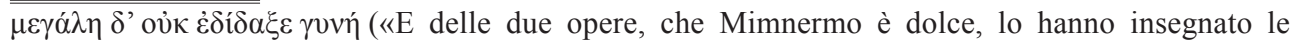
sue magnifiche usignole, non la sua grande donna».

28 Per l'identificazione della $\mu \varepsilon \gamma \alpha \dot{\alpha} \lambda \eta \gamma v v \eta ́$ con la Smirneide, cfr. CASANOva 2015, p. 147, cui rimando per ulteriori indicazioni bibliografiche.

29 Come illustrato da CANOBBio 2008, p. 187 e ss., non manca in Marziale la polemica, tipicamente callimachea, contro l'epica come grande opus (si veda, ad esempio, l'epigramma 9, 10). In tal modo, le dimensioni antitetiche del breve e del longum convivono nell'opera di Marziale contribuiscono alla sua «calcolata instabilità», volta a soddisfare un pubblico il più possibile vasto ed eterogeneo.

30 L'ipotesi, che risale a WeICHERT 1830, p. 256 e HAUPT 1866, pp. 39-40, è recentemente ribadita da Courtney 1993, p. 300; Merli 2015, p. 382 nt. 32; Hollis 2007, p. 306. Shackleton Bailey 1993, p. $301 \mathrm{nt}$. b si limita a osservare che «it is strange that Martial chose this work as an example of a long epic poem». BYRNe 2004, p. 262 ipotizza che l'Amazonis potesse essere un epillio dedicato a Pentesilea (tale genere, tuttavia, non prevede un'articolazione in più libri); secondo FogAzzA 1981, p. 24 si tratterebbe invece di una vasta raccolta di componimenti rivolti alla donna amata; per CAMERON 1995, p. 312 di un'opera elegiaca, sul modello della Smirneide di Mimnermo. Alfonsi 1942-1943, p. 82 proponeva addirittura (senza fondate ragioni) di identificare l'Amazonis con un'altra opera di Marso, le Fabellae, di cui possediamo un solo frammento (fr. 2 Blänsdorf $=$ Courtney $=175$ Hollis). Sulla possibilità di cogliere un riferimento a un'opera di Marso sulle Amazzoni in Hor. Carm. 4, 4, 17-22 (Videre Raetis bella sub Alpibus / Drusum gerentem Vindelici, quibus / mos unde deductus per omne / tempus Amazonia secure / dextras obarmet, quaerere distuli / nec scire fas est omnia) vd. BYRNE 2004, p. 261; l'ipotesi era già esclusa da BARdON 1956, p. 57; MARiotTi 1963, p. 596 nt. 27; FogAZZA 1981, p. 23. 
tentativo non molto riuscito, da parte dell'anziano epigrammista, di sperimentare un genere poetico troppo impegnativo. ${ }^{31}$

Il riferimento esplicito alla levitas, tuttavia, sembrerebbe far pensare che l'Amazonis appartenesse a un genere poetico ben distante dalla gravitas dell'epica. Marso, inoltre, non è mai annoverato dalle fonti antiche tra gli epici di età augustea ${ }^{32} \mathrm{e}$, anzi, gli altri riferimenti presenti all'interno dell'opera di Marziale sembrano giocare insistentemente sulla contrapposizione tra la poesia levis di Marso e l'epica grandiosa del cothurnatus Virgilio. Marso è presentato, insomma, come autore "anti-epico", al quale Marziale preferisce accostarsi non solo per modestia ma anche per l'orgogliosa adesione a un genus inferius di poesia. ${ }^{33}$ Nello stesso epigramma 4, 29, Marziale sembrerebbe identificarsi ancora una volta con Marso, esprimendo la preoccupazione che la sua opera potesse ottenere un simile insuccesso: tale paragone non avrebbe avuto molto senso se Marziale avesse confrontato la sua poesia epigrammatica con un'opera epica, impegnativa ed estesa per definizione. ${ }^{34}$ Anche l'allusione alla satira di Persio risulterebbe senz'altro più calzante se rapportata a un genere affine e ugualmente "minore", come l'epigramma.

È forse possibile ipotizzare, pertanto, che l'Amazonis fosse una raccolta epigrammatica particolarmente ampia ${ }^{35} \mathrm{e}$ che, proprio per questo, non avesse incontrato il favore del lector gulosus di fede callimachea.

La contrapposizione con Virgilio è esplicitata nell'epigramma 5, 5:

Sexte, Palatinae cultor facunde Minervae, ingenio frueris qui propiore dei -

nam tibi nascentes domini cognoscere curas et secreta ducis pectora nosse licet -:

5 sit locus et nostris aliqua tibi parte libellis, qua Pedo, qua Marsus quaque Catullus erit.

Ad Capitolini caelestia carmina belli grande cothurnati pone Maronis opus.

Marziale chiede a Sesto, probabilmente bibliotecario o segretario personale di Domiziano, di trovare un posto per i suoi libelli nella biblioteca imperiale. ${ }^{36}$ È questo

\footnotetext{
$\overline{\overline{31} \text { Secondo MARIOTTI }}$ 1963, p. 596 nt. 26 «per Marso, come per Virgilio e secondo una vecchia tradizione, il poema epico fu opera della maturità».

32 L'unica testimonianza che consentirebbe di ricondurre Marso a un contesto epico è Ov. Pont. 4, 16, 5, in cui è citato insieme a Rabirio, all'Iliacus Macro e al sidereus Pedone. Tuttavia, non si tratta propriamente di un catalogo di autori epici, ma soltanto di un elenco, non ordinato, dei grandi autori attivi a Roma prima che Ovidio fosse esiliato. Non è certo impossibile che Marso si fosse cimentato anche nel genere epico, come del resto fece lo stesso Pedone, autore di epigrammi e di epos, ma non è affatto certo che la sua eventuale produzione epica si possa identificare con la perduta Amazonis.

33 Cfr. Fogazza 1981, p. 25.

34 Cfr. Henriksén 2019, p. 463.

35 L'ipotesi è accennata da Schмidt 1967, p. 1580: «Martial, der sich 4, 29 für die Schar seiner libelli entschuldigt, wird deshalb, wenn er die Amazonis des D. von dem unus liber des Persius abhebt, eher auf eine Epigrammsammlung in meheren zum Beispiel als auf ein Epos, wie die communis opinio will, anspielen» e da Fogazza 1981, p. 24. È ripresa recentemente da HenRIKsén 2019, p. 461 e ss.

36 Per un commento approfondito all'epigramma 5, 5 si veda CANOBBIO 2011, p. 107 e ss.
} 
un momento molto importante nel percorso che conduce l'autore verso una progressiva presa di coscienza del valore della propria opera, non più adatta soltanto a essere recitata durante le feste o sfogliata distrattamente, ma degna di essere letta al pari delle opere dei più grandi poeti latini. Anche se, specifica Marziale, in una nota encomiastica, è opportuno che i caelestia carmina di Domiziano ${ }^{37}$ siano collocati altrove, a fianco del grande opus dell'inarrivabile Virgilio.

Di tono affine è 1'epigramma 7,99, indirizzato questa volta al liberto Crispino, al quale si chiede di sussurrare nelle orecchie dell'imperatore che Marziale nec Marso nimium minor est doctoque Catullo. Marso e Catullo, ancora una volta in coppia, non sono più dei modelli ai quali Marziale osa accostarsi solo timidamente e non sono neppure impiegati soltanto per legittimare la lasciva verborum veritas o la lunghezza degli epigrammi: la grande fama ormai raggiunta, anche presso l'imperatore in persona, fa sì che il poeta non debba più giustificarsi e possa anzi ambire, senza suscitare scalpore, a essere annoverato fra i più grandi, per quanto all'interno di un genere poetico "minore", cui il poeta sembra voler dichiarare eterna fedeltà.

Nell' epigramma 7, 29, Marziale si rivolge al giovane amato da Voconio Vittore, suo patrono e poeta, sperando che possa convincerlo ad accettare le sue poesiole, proprio come Mecenate, pur ascoltando il sublime canto di Virgilio per Alessi, non disdegnava neppure Domizio Marso, con la sua fusca Melaenis:

Thestyle, Victoris tormentum dulce Voconi, quo nemo est toto notior orbe puer,

sic etiam positis formosus amere capillis et placeat vati nulla puella tuo:

5 paulisper domini doctos sepone libellos, carmina Victori dum lego parva tuo.

Et Maecenati, Maro cum cantaret Alexin, nota tamen Marsi fusca Melaenis erat.

Vittore è avvicinato allo stesso tempo a Mecenate e a Virgilio, mentre Marziale si accosta ancora una volta a Marso. Oltre alla comune fede epigrammatica, i due autori sono legati da una forte affinità del contesto storico: ${ }^{38}$ il poeta che vive in piena età imperiale e che si impegna costantemente per ottenere il sostegno di potenti protettori, non può non riconoscersi, infatti, in un poeta della corte augustea e, in particolare, nell'epigrammista Domizio Marso, autore di poesie leggere, molto distanti dall' epos virgiliano, ma non per questo indegne di essere ascoltate. Marso stesso, del resto, in

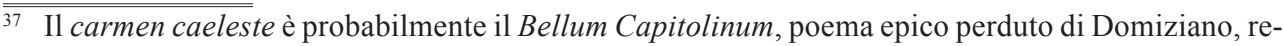
lativo all'assedio del Campidoglio da parte dei Vitelliani durante la fase finale della guerra civile del 69 d.C., su cui si vedano Barzanò 1982, pp. 11-20 e Penwill 2000, pp. 60-83.

38 Cfr. CANOBBIo 2005, pp. 157-158: Marziale si identifica con Marso in quanto "epigrammista mecenaziano" e lo fa all'interno di un disegno più ampio che percorre tutto l'ottavo libro e che è volto a suggerire «per sé e per Domiziano, suo destinatario designato, una identità augustea: lo scopo di questo atteggiamento comunicativo si lascia facilmente individuare in un invito al mecenatismo, a una politica di sostegno dei migliori esponenti della cultura letteraria del tempo fra i quali Marziale ritiene ormai di poter essere annoverato».
} 
uno dei suoi componimenti più significativi, ${ }^{39}$ piange la morte, avvenuta a breve distanza, di Tibullo e di Virgilio, che considera parimenti grandi, per quanto uno abbia cantato elegis molles amores, mentre l'altro forti regia bella pede.

Non sappiamo nulla della fusca Melaenis di Marso, di cui non possediamo alcun frammento. È possibile che Melaenis e Amazonis fossero nomi di donna ${ }^{40}$ e che, come la Lyde di Antimaco, la Leontion di Ermesianatte, la Bittis di Filita o la Cynthia di Properzio, suggellassero delle raccolte di componimenti di argomento amoroso. Non sappiamo se si trattasse di opere elegiache (di cui non possediamo alcuna notizia) oppure, basandosi soltanto sulle testimonianze di Marziale, di ampie raccolte di epigrammi (forse anche epigrammata longa). Marso non sarebbe, in questo caso, soltanto il sagace autore di epigrammi brevi, secondo quanto da lui stesso teorizzato nel perduto trattato De urbanitate, ${ }^{41}$ ma anche di impegnative raccolte di componimenti di natura erotica, forse vicini al modello elegiaco. ${ }^{42}$

\footnotetext{
$\overline{\overline{39} \text { Fr. } 7 \text { Blänsdorf }}=$ Courtney $=180$ Hollis $)$.

40 Amazonis è attestato come nome di donna nelle seguenti iscrizioni greche: $I G$ XII, Suppl. 439; $M A$ $M A$ VII, 520 (= CIG II 2768, già notato da FogazZA 1981, p. 24); SEG VII, 1073; TAM III, 1, 579. Come nome maschile in SEG XXXVI, 970; TAM III, 1, 230; IGL Syr 13, 1, 9407. In lingua latina si riscontra il dato onomastico Amazonius per soggetti di sesso maschile (ad esempio CIL V, 4057 e VI, 11521), il cognome Amazon (ad esempio CIL vI, 11131 e 32508) e Amazonia per personaggi femminili (CIL VI, 3451). Il termine Amazonis, inoltre, ha anche un impiego specifico nella poesia erotica: in Ov. ars 2, 743 e 3, 1, l'Amazzone indica, per antonomasia, la donna che rifiuta l'amore, costringendo l'uomo a intraprendere una dura battaglia, simile allo scontro mitico tra i Greci e le Amazzoni (si veda il commento di GiBSON 2003, p. 86). È possibile che a questo significato alludesse anche Marso nella sua raccolta di componimenti amorosi. Il nome Melaenis, invece, è attestato in greco e allude a una caratteristica fisica: la pelle scura. In Mart. 7, 29, 8 è possibile riconoscere un gioco di parole nell'accostamento di Melaenis con l'aggettivo fusca, che ha il medesimo significato. Si tratta anche di un epiteto di Afrodite a Corinto (vd. RE 15.1, s.v. Melaina, coll. 384-385) e del nome di un personaggio della Cistellaria plautina. Come osserva LuCK 1959, p. 56, «lovely dark girls are often praised in the erotic epigrams of Book V of the Greek Anthology»: è possibile che, allo stesso modo, Marso avesse dedicato all'amata Melenide un'opera epigrammatica o un ciclo di epigrammi (cfr. FogAzZA 1981, p. 17). È stato identificato, inoltre, un riferimento intertestuale alla seconda ecloga virgiliana, in cui Alessi è definito candidus $(2,16)$ e paragonato al niger Menalca: la carnagione scura, percepita quasi come un difetto fisico (cfr. Ov. ars 3, 270; Mart. 3, 34, 2; 4, 62, 1; 6, 39, 18; 7, 13, 2), potrebbe designare, nel confronto con Virgilio, il carattere "minore" dell'opera di Marso e di Marziale. Sull'argomento si vedano Galán Vioque - Zoltowski 2002, p. 213; Vallat 2008, p. 157 e Moreno Soldevila - Marina Castillo - Fernández Valverde 2019, pp. 387-388.

${ }_{41} \mathrm{Nel}$ frammento tradito da Quint. inst. 6, 3, 104, Marso definisce "urbanitas" quaedam in breve dictum coacta et apta ad delectandos movendosque homines in omnem adfectum animi, maxime idonea ad resistendum vel lacessendum, prout quaeque res ac persona desiderat. RAMAGE 1959, p. 254 ha verosimilmente ipotizzato che il trattato altro non fosse che una riflessione teorica sul genere poetico praticato da Marso, ossia l'epigramma. È possibile che il De urbanitate sia stato una fonte importante per l'elaborazione dell'epigramma marzialiano, come suggerisce CiтRONI 2015, p. 83.

42 Il fatto che il levis Marso fosse anche autore di opere particolarmente ponderose potrebbe essere confermato dalla testimonianza di Char. $91 \mathrm{~B}(72 \mathrm{~K})$, che cita il frammento 2 Blänsdorf (= Courtney = 175 Hollis) attribuendolo al nono libro delle Fabellae. Contrario a questa interpretazione (sostenuta, tra gli altri, da Hollis 2007, p. 308) è HeNRIKSÉN 2019, p. 470 nt. 2, secondo cui il numero viIII, riportato dai codici di Carisio, alluderebbe piuttosto al nono componimento della raccolta.
} 
La progressiva identificazione di Marziale con Domizio Marso trova coronamento nell'epigramma 8, 55 (56), non a caso, proprio un epigramma longum:

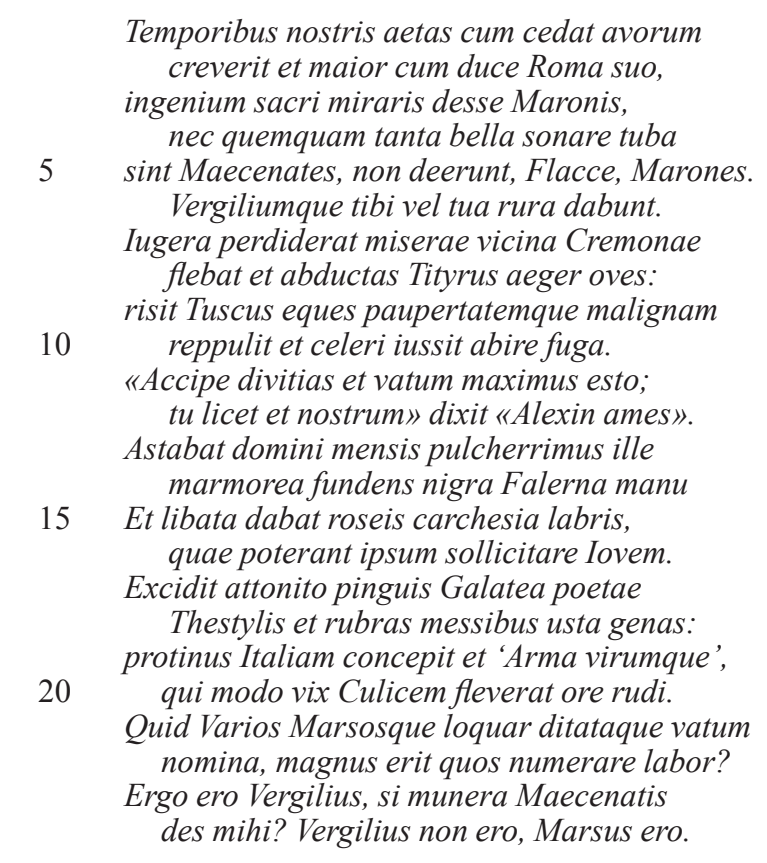

Dopo aver lamentato l'assenza, ai suoi tempi, di grandi autori come Virgilio, imputandola alla mancanza di protettori delle arti, Marziale conclude che comunque, se anche vi fosse un nuovo Mecenate, egli non sarebbe un Virgilio ma un Marso.

Con queste parole si attua la completa sovrapposizione con il poeta della corte augustea, che Marziale vede come il proprio modello principale, non solo nella composizione di un epigramma urbanus, eventualmente longus, ma anche nel rapporto con il potere. Marso incarna, non meno di Virgilio, l'ideale di una piena e spontanea collaborazione tra il letterato e il princeps, alla quale Marziale non fa che aspirare nel corso di tutta la sua carriera.

Dall'analisi delle testimonianze marzialiane, possiamo dedurre pertanto che Marso, prendendo le mosse dal modello catulliano, non esitò a ricercare soluzioni poetiche differenti, caratterizzate dalla commistione di vari generi letterari e da un forte sperimentalismo, nelle forme e nei contenuti: fu ora poeta leggero e ora impegnato, offrendo a Marziale un modello fondamentale, sia nell'interpretazione fluida e aperta del genere epigrammatico, sia per la capacità dell'epigramma di adempiere, non meno della poesia epica, a un compito di celebrazione del potere. A questo compito Marziale non intendeva assolutamente sottrarsi, pur mantenendosi fedele, come fu probabilmente Domizio Marso, alla sua Musa minore. 


\section{RIFERIMENTI BIBLIOGRAFICI}

Alfonsi 1942-1943 : L. Alfonsi, Sulla poesia di Domizio Marso, «Atti e Mem. Accad. Sc. Lett. ed Arti Padova» 59 (1942-1943), 344, pp. 77-85.

Alfonsi 1964 : L. Alfonsi, Incontri: Domizio Marso, «Maia» 16 (1964), pp. 377-388.

Alfonsi 1966 : L. Alfonsi, Sull'epigramma di Cicerone, «Rivista di Filologia e di Istruzione Classica» 94 (1966), pp. 302-303.

Avery 1960 : W. T. Avery, The Year of Tibullus' Death, «The Classical Journal» 55 (1960), 5, pp. 205-209.

BARDON 1956 : H. Bardon, La littérature latine inconnue, II, L'Epoque impériale, Paris, Klincksieck, 1956.

Barigazzi 1964 : A. Barigazzi, Su due epigrammi di Domizio Marso, «Athenaeum» 42 (1964), pp. 261-268.

Barrett 1996 : A. A. Barrett, Agrippina: Sex, Power, and Politics in the Early Empire, New Haven-London, Yale University Press, 1996.

BArzanò 1982 : A. Barzanò, Domiziano e il "Bellum Capitolinum”, «Rendiconti. Classe di lettere e scienze morali e storiche. Istituto Lombardo. Accademia di scienze e lettere» 116 (1982), pp. 11-20.

BARZANÒ 2011 : A. Barzanò, La politica dinastica di Caligola e la cosiddetta congiura del 39 d.C., «Aevum» 85 (2011), pp. 65-80.

BläNSDORF 2011: J. Blänsdorf(ed.), Fragmenta Poetarum Latinorum Epicorum et Lyricorum praeter Enni Annales et Ciceronis Germanicique Aratea post Wilhelm Morel et Karl Büchner ed. quartam auctam, Berlin-New York, De Gruyter, 2011 (I ed. 1995).

BoRGO 2003 : A. Borgo, Levis nel dibattitto critico-letterario romano, in Tra strategie retoriche e generi letterari. Dieci studi di letteratura latina, a cura di V. Viparelli, Napoli, Liguori, 2003, pp. 1-14.

Byrne 2004 : S. N. Byrne, Martial's fiction: Domitius Marsus and Maecenas, «The Classical Quarterly» 54 (2004), pp. 255-265.

Cameron 1995 : A. Cameron, Callimachus and his Critics, Princeton, Princeton University Press, 1995.

CAnobBio 2005 : A. Canobbio, Il libro VIII di Marziale e la ricerca di una identità augustea, in Modelli letterari e ideologia nell'età Flavia, atti della III giornata ghisleriana di Filologia classica (Pavia 2003), a cura di F. Gasti - G. Mazzoli, Como-Pavia, Ibis, 2005, pp. 127-162. 
CAnobBio 2008 : A. Canobbio, Epigrammata longa e breves libelli. Dinamiche formali dell'epigramma marzialiano, in Epigramma longum. Da Marziale alla tarda antichità, atti del convegno (Cassino 2006), a cura di A. M. Morelli, I-II, Cassino 2008, I, pp. 69-193.

CAnobBio 2011 : A. Canobbio (ed.), M. Valerii Martialis Epigrammaton liber quintus, Napoli, Loffredo, 2011.

CASAnova 2015 : A. Casanova, Leggere oggi i primi versi del Prologo degli Aitia, «Prometheus» 41 (2015), pp. 141-154.

CIG : Corpus Iscriptionum Graecarum.

CIL : Corpus Inscriptionum Latinarum.

Citroni 1991 : M. Citroni, L'epigramma, in F. Montanari, La poesia latina. Forme, autori, problemi, Roma, NIS, 1991, pp. 171-189, 203-207.

Citroni 1995 : M. Citroni, Poesia e lettori in Roma antica. Forme della comunicazione letteraria, Roma-Bari, Laterza, 1995.

Citroni 2004 : M. Citroni, Martial, Pline le jeune, et l'identité de genre de l'épigramme latine, «Dictynna» 1 (2004), pp. 125-153.

Citroni 2015 : M. Citroni, Marziale e la tradizione dell'epigramma latino, in MerLi 2015 (I ed. 1996), pp. 65-106.

Courtney 1993 : E. Courtney (ed.), The Fragmentary Latin Poets, ed. by E. Courtney, Oxford, Clarendon Press, 1993.

Cozzolino 1986 : A. Cozzolino, Trent'anni di studi sui poeti epici minori d'età augustea (19561985), «Vichiana» 15 (1986), pp. 237-244

Cozzolino 1992 : A. Cozzolino, Sul frammento di Albinovano Pedone, in Miscellanea di studi in onore di Armando Salvatore, a cura di E. Flores, A.V. Nazzaro, L. Nicastri, G. Polara, Napoli, Loffredo, 1992, pp. 109-120.

Dahlmann 1975 : H. Dahlmann, Zum Fragment des Albinovanus Pedo (Mor. S. 115), in H. Dahlmann, Cornelius Severus, Mainz, Akademie der Wissenschaften und der Literatur, 1975, 6, pp. 128-137.

Dahlmann 1979 : H. Dahlmann, Das Fragment des Gn. Cornelius Lentulus Gaetulicus (FPL Morel S. 123), in Studi in onore di Antonio Traglia, I-II, Roma, Edizioni di storia e letteratura, 1979, II, pp. 657-667.

Duret 1983 : L. Duret, Dans l'ombres des plus grands, «ANRW» II, 32/2, Berlin-New York, De Gruyter, 1983, pp. 1447-1560.

Fogazza 1981 : D. Fogazza (ed.), Domiti Marsi testimonia et fragmenta, Roma, Edizioni di Storia e Letteratura, 1981.

Formicola 2009 : C. Formicola, Poetica dell'«imitatio» e funzione del modello: Properzio nei versi di Sidonio Apollinare, «Voces» 20 (2009), pp. 81-101. 
Galán Vioque - Zoltowski 2002 : G. Galán Vioque - J. J. Zoltowski (edd.), Martial Book VII. A Commentary, Leiden, Brill, 2002.

Gibson 2003 : R. K. Gibson (ed.), Ovid. Ars Amatoria, Book 3, Cambridge, Cambridge University Press, 2003.

Haupt 1876 : M. Haupt, Opuscola III, Lipsiae, Hirzel, 1876.

Havet 1900 : L. Havet, Domitius Marsus: sur Bavius et son frére, «Revue de Philologie, de Littérature et d'Histoire Anciennes» 24 (1900), pp. 89-96.

HENRIKSÉN 2019 : C. Henriksén, Latin Epigram in the Early Empire, in A Companion to Ancient Epigram, ed. by C. Henriksén, Hoboken, Wiley Blackwell, pp. 459-474.

Hollis 2007 : A. S. Hollis (ed.), Fragments of Roman Poetry c. 60 B. C. - A. D. 20, Oxford, Oxford University Press, 2007.

Hubaux 1930 : J. Hubaux, Les thèmes bucoliques dans la poésie latine, Bruxelles, Maurice Lamertin, 1930.

$I G:$ Inscriptiones Graecae.

Lombardi 1977 : L. Lombardi, A proposito di alcuni recenti studi su Domizio Marso, «Boll. Stud. Lat.» 7 (1977), pp. 343-358.

Luck 1959 : G. Luck, The Latin Love Elegy, London, Methuen, 1959.

Malcovati 1923 : E. Malcovati, De Gaetulico Graecorum epigrammatum scriptore, «Athenaeum» 1 (1923), pp. 32-38.

MAMA : Monumenta Asiae Minoris Antiqua.

MARIOTti 1963 : S. Mariotti, Intorno a Domizio Marso, in Miscellanea di Studi Alessandrini in memoria di Augusto Rostagni, Torino, Bottega d'Erasmo, 1963, pp. 588-614.

Mazzoli 2002 : G. Mazzoli, Il lamento dell'ignoto marinaio (ancora sul v. 19 di Albinovano Pedone), in Curiositas: studi di cultura classica e medievale in onore di Ubaldo Pizzani, a cura di E. Menestò, A. Di Pilla, A. Isola, Napoli, Edizioni Scientifiche Italiane, 2002, pp. 167-177.

Merli 2015 : E. Merli (ed.), Marco Valerio Marziale. Epigrammi, saggio introduttivo di M. Citroni, traduzione di M. Scandola, note di E. Merli, Milano, Biblioteca Universale Rizzoli, 2015 (I ed. 1996).

Merli - Romano 2017 : E. Merli-E. Romano, La letteratura tiberiana: prospettive di ricerca, «Vichiana» 54, 2 (2017), pp. 37-56.

Moreldi 2008a : A. M. Morelli, Epigramma longum: in cerca di una básanos per il genere epigrammatico, in Epigramma longum. Da Marziale alla tarda antichità, atti del convegno (Cassino 2006), a cura di A. M. Morelli, Cassino, Edizioni dell'Università degli Studi di Cassino, 2008, pp. 17-51. 
Morelli 2008b : A. M. Morelli, Gli epigrammi erotici "lunghi” in distici di Catullo e Marziale. Morfologia e statuto di genere, in Epigramma longum. Da Marziale alla tarda antichità, Atti del Convegno Internazionale (Cassino 2006), a cura di A. M. Morelli, Cassino, Edizioni dell’Università degli Studi di Cassino, 2008, pp. 81-130.

Moreno Soldevila 2006 : R. Moreno Soldevila (ed.), Martial. Book IV. A Commentary, LeidenBoston, Brill, 2006.

Moreno Soldevila - Marina Castillo - Fernàndez Valverde 2019 : R. Moreno Soldevila - A. Marina Castillo - J. Fernàndez Valverde, A Prosopography to Martial's Epigrams, Berlin-Boston, De Gruyter, 2019.

Munari 1955 : F. Munari (ed.), Epigrammata Bobiensia, II, Introduzione ed edizione critica, Roma, Edizioni di Storia e Letteratura, 1955.

Nauta 2007 : R. R. Nauta, Literary History in Martial, in Dialogando con il passato. Permanenze e innovazioni nella cultura latina di età Flavia, a cura di A. Bonadeo - E. Romano, Firenze, Le Monnier, 2007, pp. 1-17.

Page 1981 : D. L. Page (ed.), Further Greek Epigrams, Cambridge, Cambridge University Press, 1981.

Paratore 1964 : E. Paratore, Ancora su Domizio Marso, «Rivista di Cultura Classica Medievale» 6 (1964), pp. 64-96.

Pasetto - Sansone 2016 : C. Pasetto - A. Sansone, Un Domitius alla corte di Augusto: frammenti della vita e dell'opera di Domizio Marso, «Sibrium» 30 (2016), pp. 155-185.

Penwill 2000 : J. L. Penwill, Quintilian, Statius and the Lost Epic of Domitian, «Ramus» 29 (2000), pp. 60-83.

Pfeiffer 1949 : R. Pfeiffer (ed.), Callimachus, I, Fragmenta, Oxford, Clarendon Press, 1949.

Pianezzola 1984 : E. Pianezzola, Au delà des frontières du monde: Un topos rhétorique pour un rétablissement du texte d'Albinovanus Pédo (p. 116 Mor.=148 Buechn. v. 19), «REL» 62, 1984, pp. 192-205.

Ramage 1959 : E. S. Ramage, The “De Urbanitate” of Domitius Marsus, «Classical Philology» 54 (1959), pp. 250-255.

RibBECK 1889 : O. Ribbeck, Geschichte der römischen Dichtung, II, Stuttgard, Cotta, 1889.

Rocca 1989 : R. Rocca, Epici minori d'età augustea, Genova, Dipartimento di Archeologia, Filologia classica e loro tradizioni, 1989.

Rohden 1894 : P. Rohden, s.v. Albinovanus n.5, in RE i 1 (1894) col. 1314.

Rostagni 1964 : A. Rostagni, Storia della letteratura latina, I-III, Torino, Utet, 1964 (I ed. 1936).

Schmidt 1967 : P. L. Schmidt, s.v. Domitius Marsus, in Kl. Pauly 2 (1967) pp. 1579-1580.

SEG : Supplementum Epigraphicum Graecum. 
Shackleton Bailey 1993 : D. R. Shackleton Bailey (ed.), Martial. Epigrams, i, Cambridge (Mass.)-London, Harvard University Press, 1993.

Skutsch 1905 : F. Skutsch, s.v. Domitius Marsus, in RE v 1, 1903, pp. 1430-1432.

Squillante Saccone 2009 : M. Squillante Saccone, La biblioteca di Sidonio Apollinare, «Voces» 20 (2009), pp. 139-159.

Stein - Skutsch 1900 : E. Stein - F. Skutsch, s.v. Cornelius n. 220, in RE IV, 1 (1900) coll. 1384-1386.

Szelest 1980 : H. Szelest, Ut faciam breviora mones epigrammata, Corde ... Eine MartialStudie, «Philologus» 124 (1980), pp. 99-108.

TAM : Tituli Asiae Minoris.

TANDOI 1964 : V. Tandoi, Albinovano Pedone e la retorica giulio-claudia delle conquiste, «SIFC» 36 (1964), pp. 129-168.

TANDOI 1967 : V. Tandoi, Albinovano Pedone e la retorica giulio-claudia delle conquiste, «Studi italiani di filología classica», 39 (1967), pp. 5-66.

Tandor 1985 : V. Tandoi, Disiecti Membra Poetae. Studi di poesia latina in frammenti, II, Foggia, Atlantica, 1985.

Traglia 1987 : A. Traglia, Poeti latini dell'età giulio-claudia misconosciuti, I. Domizio Marso, «Studi italiani di filologia classica» 26 (1987), pp. 44-53.

Traglia 1988 : A. Traglia, Cn. Cornelio Lentulo Getulico e il suo frammento esametrico, «Cultura e Scuola» 27 (1988), pp. 56-61.

Traina 1985 : A. Traina, Dal Morel al Büchner. In margine alla nuova edizione dei Fragmenta poetarum Latinorum, «Rivista di Filologia e di Istruzione Classica» 113 (1985), pp. 96-119.

Vallat 2008 : D. Vallat, Onomastique, culture et société dans les Épigrammes de Martial, Bruxelles, Latomus, 2008.

Weichert 1830 : J. A. Weichert, Poetarum latinorum Hostii, Laevii, C. Licinii Calvi, C. Helvii Cinnae, C. Valgii Rufi, Domitii Marsi aliorumque vitae et carminum reliquiae, Lipsiae, Teubner, 1830.

Williams 2004 : C. A. Williams (ed.), Martial, Epigrams, Book Two, Edited with Introduction, Translation, and Commentary, Oxford, Oxford University Press, 2004. 